\title{
Robust Output Control of an Uncertain Underactuated 2DOF Mass-Spring-Damper System with Backlash Based on Active Disturbance Rejection Control Structure
}

\author{
C. Mario Mora, ${ }^{1}$ A. David Rosas $\mathbb{D},{ }^{1}$ C. Raul Rascon, ${ }^{1}$ and G. Ricardo Cuesta $\mathbb{D}^{2}$ \\ ${ }^{1}$ Facultad de Ingeniería, Universidad Autónoma de Baja California, Blvd Benito Juárez S/N, Mexicali, BC, Mexico \\ ${ }^{2}$ Electronics Department, Ensenada Center for Scientific Research and Higher Education, Ensenada, BC, Mexico \\ Correspondence should be addressed to A. David Rosas; drosas@uabc.edu.mx
}

Received 7 December 2017; Accepted 10 June 2018; Published 5 July 2018

Academic Editor: Achille Paolone

Copyright @ 2018 C. Mario Mora et al. This is an open access article distributed under the Creative Commons Attribution License, which permits unrestricted use, distribution, and reproduction in any medium, provided the original work is properly cited.

\begin{abstract}
We propose a strategy to solve the tracking and regulation problem for a $2 \mathrm{DOF}$ underactuated mass-spring-damper system with backlash on the underactuated joint, parametric uncertainties, and partial measurement of the state vector. The design of the controller is divided into two stages; in the first stage, it is assumed that the full state vector and all perturbations in the system are available. The model is divided into one actuated subsystem and one underactuated subsystem. The position of the actuated mass is defined as the control input of the underactuated subsystem, which is designed as an ideal controller that solves the tracking and regulation control problems for the underactuated mass. Finally, the control input of the actuated subsystem is designed to solve the tracking problem considering as a reference signal the control signal of the underactuated subsystem. The second stage solves the problem of the implementation of the previously designed ideal controller using the active disturbances rejection control structure (ADRC). Here state observers estimate the nonmeasured state variables and, at the same time, estimate perturbations and auxiliary signals for their compensation. The performance of the closed-loop system is illustrated by numerical simulations and experimental results.
\end{abstract}

\section{Introduction}

Control of underactuated mechanical systems, mechanisms with fewer number of control inputs than their degrees of freedom, has received much attention in the last decades. This is because of the theoretical challenges as well as practical applicability; robots, aerospace vehicles, underwater vehicles, and surface vessels are some examples of underactuated mechanical systems $[1,2]$. While many important techniques and results have been presented for this class of systems, the control of them remains an open problem when we considering several practical situations like disturbances in the plant, partial measurement of the state vector, and the presence of hard nonlinearities like dry friction and backlash; important issues are how control models can be formulated for such systems and how closed-loop control problems can be solved and implemented [3].

Backlash is a phenomenon that limits the performance of the control systems; it introduces errors in stable state; it even may produce limit cycles. Backlash has been studied since the 1940s and many proposals have appeared to mitigate its effects [4]. Different ways exist to model the backlash phenomenon; in [5], it is established that backlash and hysteresis are different phenomena; however, many times they are used the same; also dead zone functions [6], differential equations [7], and smooth approximations of the dead zone function have been used $[8,9]$. However, as far as we know, there is no consensus about a unique backlash model; therefore, many papers use the model that allows solving a specific control problem, as we do in the present work.

Some control algorithms that solve regulation and tracking control objectives for underactuated mechanical systems, without considering practical issues, are proposed in [10-12] and references therein. Also, there are papers that propose control algorithms that produce a closed-loop system with some degree of robustness, for example, [13, 14].

Two works that address the control problem of the underactuated systems with backlash are $[15,16]$. In [15], an 


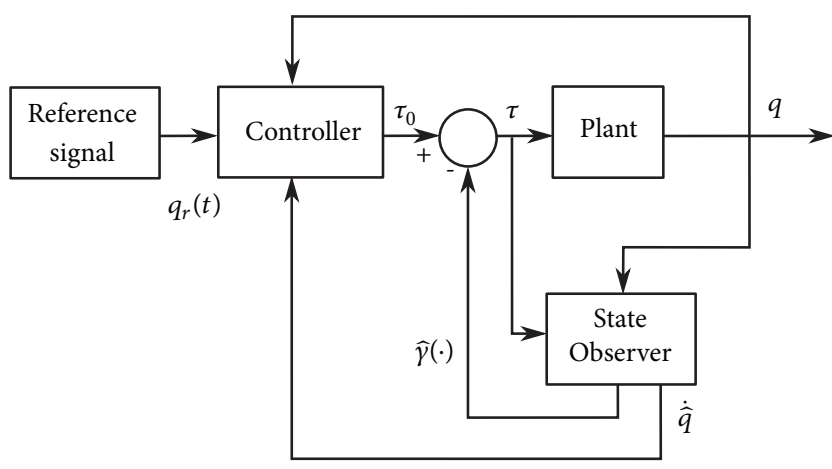

FIGURE 1: A block diagram of the active disturbance rejection control structure.

$H_{\infty}$ control for tracking of a class of nonsmooth systems that include dry friction and backlash is proposed. In this case, a modified version of the $H_{\infty}$ control achieves a good performance for tracking on a system called Industrial Emulator, model 220 of Educational Control Products. However, it cannot be applied to solve the regulation problem because a necessary condition is that the derivative of the reference signal must be different from zero for almost all times. In [16], a controller based on $H_{\infty}$ control to solve the regulation problem on a $2 \mathrm{DOF}$ underactuated massspring-damper system with backlash and dry friction is proposed. In this case, they get satisfactory results but the strategy cannot be applied to tracking control in direct way.

An important strategy to reduce or eliminate the effect produced by disturbances and parametric uncertainties in closed-loop system, for fully actuated mechanical systems, is the active disturbances rejection control (ADRC) shown in Figure 1. This control structure includes a robust observer to estimate the velocity vector, perturbations, and terms generated by parametric uncertainties; the estimated perturbations are included in the controller to eliminate actual perturbation in plant. Ideally, if the estimation is precise, the control structure guarantees, in steady state, a zero error in the control objective using a well-known continuous controller for a plant without disturbances, obtaining a robust closedloop system. The theoretical framework, stability proofs, and some applications of this control structure can be found in [17-24].

Due to good features of robustness of the ADRC control structure in full actuated systems, we hope that it can solve, under certain conditions, the regulation and tracking control problems on some class of underactuated systems.

In the present paper, we propose a control strategy to solve the tracking and regulation problem on a $2 \mathrm{DOF}$ underactuated mass-spring-damper system with backlash on the underactuated joint, parametric uncertainties, and partial measurement of the state vector, applying the basic principle of the active disturbance rejection control (ADRC) structure. Also, based on [25], we propose a smooth model of the backlash, which is formed by a lineal function and a smooth approximation of the saturation function; this function will be considered as a bounded disturbance.
The proposed control strategy is based on a simple idea: how the mass $m_{1}$ should be moved so that the mass $m_{2}$ can follow a reference signal?

The controller design is performed in two stages; in the first stage, we divide the system into one actuated subsystem and one underactuated subsystem; it is assumed that the full state vector and all perturbations in the system are available. The position of the actuated mass is defined as the control input of the underactuated subsystem, which is designed as an ideal controller that solves the tracking and regulation control problems for the underactuated mass. Finally, the control input of the actuated subsystem is designed to solve the tracking problem considering as a reference signal the control signal of the underactuated subsystem. The second stage of controller design solves the problem of the implementation of the previously designed ideal controller using the ADRC control structure. Here state observers estimate the nonmeasured state variables and, at the same time, estimate perturbations and auxiliary signals for their compensation. The performance of the closed-loop system is illustrated by numerical simulations and experimental results.

The paper's structure is as follows. In Section 2, we define the control problem and establish the model of the backlash that we use in this work. Section 3 shows the ideal control strategy where we assume the knowledge of all disturbances in the system and full measurement of state vector. In Section 4, we present the strategy to implement the ideal control based on ADRC control structure. In Section 5, we illustrate the performance of the proposed control strategy through numerical simulations and experimental results. Finally, in Section 6, we present some conclusions.

\section{Problem Statement and Preliminary Definitions}

Consider a 2DOF underactuated mass-spring-damper system, shown in Figure 2, whose model is given by

$$
\begin{aligned}
& \dot{x}_{1}=x_{2}, \\
& \dot{x}_{2}=-\frac{k_{1}}{m_{1}} x_{1}-\frac{b_{1}}{m_{1}} x_{2}+\frac{k_{2}}{m_{1}} B(\Delta x, d)+\frac{k_{a}}{m_{1}} u+\gamma_{1}(\cdot), \\
& \dot{x}_{3}=x_{4}, \\
& \dot{x}_{4}=-\frac{b_{2}}{m_{2}} x_{4}-\frac{k_{2}}{m_{1}} B(\Delta x, d)+\gamma_{2}(\cdot),
\end{aligned}
$$

where $x_{1}$ and $x_{2}$ are position and velocity of mass $m_{1}, x_{3}$ and $x_{4}$ are position and velocity of mass $m_{2}, u$ is the control input, and $B(\Delta x, d)$ is a function that describes the backlash phenomenon, whose characteristic is shown in Figure 3, where $d$ is backlash length and $\Delta x=x_{3}-x_{1} \cdot \gamma_{1}(\cdot)$ and $\gamma_{2}(\cdot)$ are disturbances due to parametric uncertainties, which are bounded if the state of the system is bounded. Finally, the parameters of the plant are the spring constants $k_{1}$ and $k_{2}(\mathrm{~N} / \mathrm{m})$ and friction constants $b_{1}$ and $b_{2}(\mathrm{~kg} / \mathrm{s})$, and $k_{a}(N / V)$ is a power amplifier constant; all parameters are positive. 


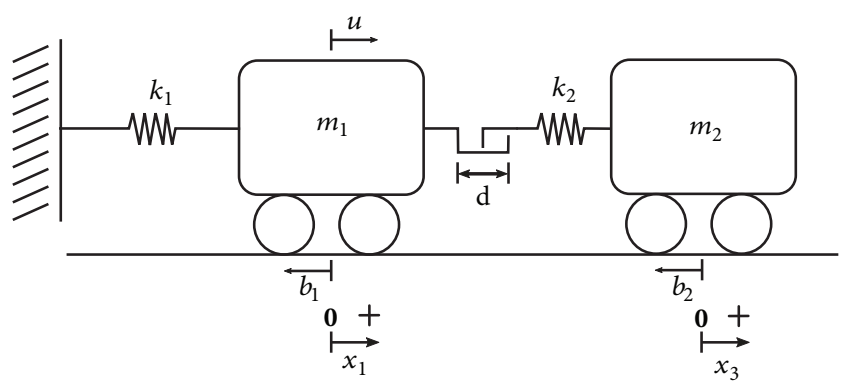

FIGURE 2: 2DOF underactuated mass-spring-damper system with backlash.

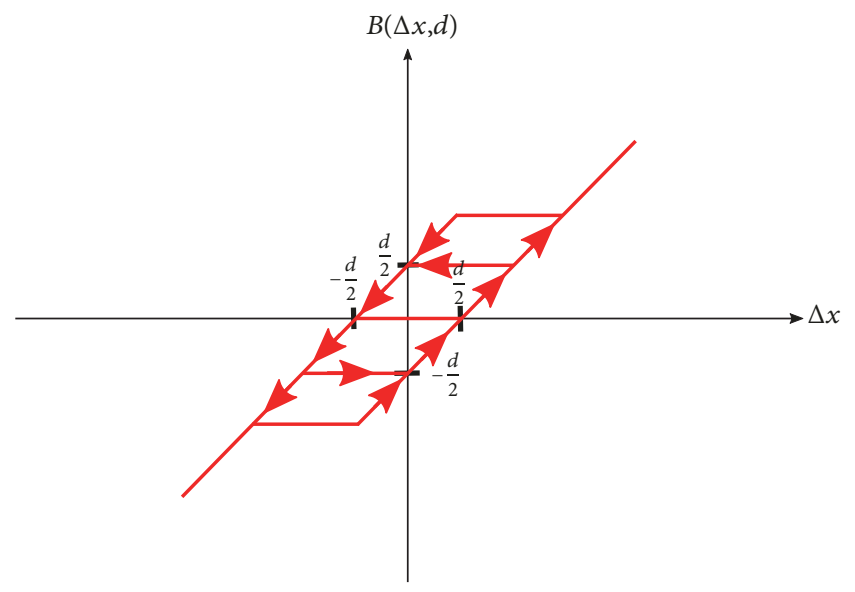

FIGURE 3: Backlash phenomenon like a hysteresis and dead zone functions combination.

To simplify the dynamic model of the system, in particular the backlash phenomenon, we use the equality proposed in [25]:

$$
B(\Delta x, d)=\operatorname{Sat} H(\Delta x, d)-\Delta x,
$$

where $\operatorname{Sat} H(\Delta x, d)$ is the saturation function with hysteresis as shown in Figure 4.

Given that the nonlinear term of function $B(\Delta x, d)$ is a collection of saturation functions $\operatorname{Sat}(\Delta x, d)$ with a displacement, which are bounded, and only one of them is active at a time, a simplified model of (2) is

$$
B(\Delta x, d)=\operatorname{Sat}(\Delta x, d)-\Delta x,
$$

where

$$
\operatorname{Sat}(\Delta x, d)=\frac{1}{2 \rho}(\log (\cosh (a))-\log (\cosh (b))),
$$

where $a=\rho(\Delta x-d / 2), b=\rho(\Delta x+d / 2)$ and $\rho=10 / d$. Function (4) is a smooth version of a saturation function and it is a $\mathscr{C}^{k}$ function. This is the model used in this work.

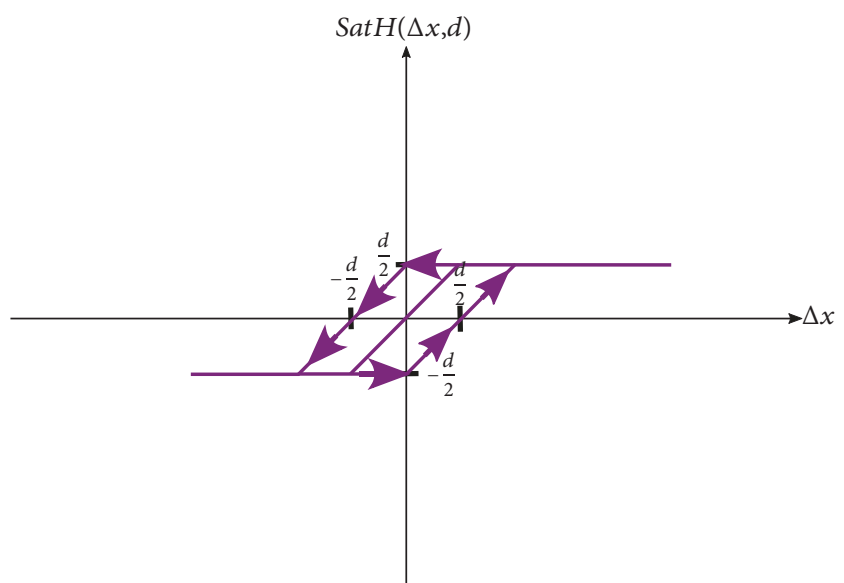

FIGURE 4: Saturation function with hysteresis $\operatorname{Sat} H(x, d)$.

Substituting (3) in (1), we have

$\dot{x}_{1}=x_{2}$,

$\dot{x}_{2}=-\frac{k_{1}+k_{2}}{m_{1}} x_{1}-\frac{b_{1}}{m_{1}} x_{2}+\frac{k_{2}}{m_{1}} x_{3}+\frac{k_{2}}{m_{1}} \operatorname{Sat}(\Delta x, d)$

$$
+\frac{k_{a}}{m_{1}} u+\gamma_{1}(\cdot)
$$

$\dot{x}_{3}=x_{4}$,

$\dot{x}_{4}=\frac{k_{2}}{m_{2}} x_{1}-\frac{k_{2}}{m_{2}} x_{3}-\frac{b_{2}}{m_{2}} x_{4}-\frac{k_{2}}{m_{2}} \operatorname{Sat}(\Delta x, d)$

$+\gamma_{2}(\cdot)$,

$y_{1}=x_{1}$

$y_{2}=x_{3}$.

The control objective is

$$
\lim _{t \rightarrow \infty}\left\|x_{3}-r(t)\right\|=0
$$

where $r(t)$ is a $\mathscr{C}^{k}$ function with sufficiently large $k$.

Now we define the error variables $e_{1}=x_{3}-r(t)$ and $e_{2}=$ $x_{4}-\dot{r}(t)$, whose dynamics are given by

$\dot{e}_{1}=e_{2}$

$\dot{e}_{2}=-\frac{k_{2}}{m_{2}} e_{1}-\frac{k_{2}}{m_{2}} e_{2}-\frac{k_{2}}{m_{2}} r(t)-\frac{b_{2}}{m_{2}} \dot{r}(t)-\ddot{r}(t)$

$$
+\Gamma(\cdot)+\frac{k_{2}}{m_{2}} x_{1}
$$

$\dot{x}_{1}=x_{2}$,

$\dot{x}_{2}=-\frac{k_{1}+k_{2}}{m_{1}} x_{1}-\frac{b_{1}}{m_{1}} x_{2}+\frac{k_{2}}{m_{1}} \operatorname{Sat}(\Delta x, d)+\frac{k_{a}}{m_{1}} u$

$+\gamma_{1}(\cdot)$ 
where

$$
\Gamma(\cdot)=-\frac{k_{2}}{m_{1}} \operatorname{Sat}(\Delta x, d)+\gamma_{2}(\cdot)
$$

is a bounded disturbance and

$$
|\Gamma(\cdot)|<\sigma .
$$

Now, the control objective is to design a control input $u$ such that the error variables tend to zero, while $x_{1}$ and $x_{2}$ stay bounded.

\section{Conceptual Solution of the Control Problem}

In this section, we propose the strategy to solve the control problem considering that all states and all disturbances are known; in the next section, we will give a strategy to implement it.

System (12) can be divided into two subsystems: an underactuated subsystem,

$$
\begin{aligned}
\dot{e_{1}}= & e_{2}, \\
\dot{e_{2}}= & -\frac{k_{2}}{m_{2}} e_{1}-\frac{b_{2}}{m_{2}} e_{2}+\Gamma(\cdot)-\frac{k_{2}}{m_{2}} r(t)-\frac{b_{2}}{m_{2}} \dot{r}(t) \\
& -\ddot{r}(t)+\frac{k_{2}}{m_{2}} x_{1},
\end{aligned}
$$

and an actuated subsystem,

$$
\begin{aligned}
\dot{x}_{1}= & x_{2}, \\
\dot{x}_{2}= & -\frac{\left(k_{1}+k_{2}\right)}{m_{1}} x_{1}-\frac{b_{1}}{m_{1}} x_{2}+\frac{k_{2}}{m_{1}} x_{3}-\frac{k_{2}}{m_{1}} \operatorname{Sat}(\Delta x, d) \\
& +\frac{k_{a}}{m_{1}} u .
\end{aligned}
$$

As we can see, the control input does not appear in subsystem (15), so we consider the state variable $x_{1}$ as a control input for it. Then, if $x_{1}=x_{1 r}$ where

$$
\begin{aligned}
x_{1 r} & =\frac{m_{2}}{k_{2}}\left(\frac{k_{2}}{m_{2}} r(t)+\frac{b_{2}}{m_{2}} \dot{r}(t)+\ddot{r}(t)-\Gamma(\cdot)-k_{p 1} e_{1}\right. \\
& \left.-k_{d 1} e_{2}\right),
\end{aligned}
$$

the origin $e_{1}=0$ and $e_{2}=0$ will be an asymptotically stable equilibrium point. Substituting (17) in (15), we obtain

$$
\begin{aligned}
& \dot{e_{1}}=e_{2}, \\
& \dot{e_{2}}=-\left(\frac{k_{2}}{m_{2}}+k_{p 1}\right) e_{1}-\left(\frac{b_{2}}{m_{2}}+k_{d 1}\right) e_{2},
\end{aligned}
$$

where $k_{p 1}$ and $k_{d 1}$ are positive constants and, given that $k_{2}, b_{2}$, and $m_{2}$ are positive constants, we guaranteed that the origin of subsystem (18) is an exponentially stable equilibrium point. is

Now, the control objective to the actuated subsystem (16)

$$
\lim _{t \rightarrow \infty}=\left\|x_{1}-x_{1 r}\right\|=0 .
$$

Define the error variables $e_{3}=x_{1}-x_{1 r}$ and $e_{4}=x_{2}-\dot{x}_{1 r}$, whose dynamics are given by

$$
\begin{aligned}
\dot{e_{3}}= & e_{4}, \\
\dot{e_{4}}= & -\frac{\left(k_{1}+k_{2}\right)}{m_{1}} e_{3}-\frac{b_{1}}{m_{1}} e_{4}+\frac{k_{2}}{m_{1}} x_{3}-\frac{\left(k_{1}+k_{2}\right)}{m_{1}} x_{1 r} \\
& -\frac{b_{1}}{m_{1}} \dot{x}_{1 r}-\ddot{x}_{1 r}-\frac{k_{2}}{m_{1}} \operatorname{Sat}(\Delta x, d)+\gamma_{1}(\cdot) \\
& +\frac{k_{a}}{m_{1}} u, \\
y_{e}= & e_{3},
\end{aligned}
$$

and then, to stabilize the origin of (20), we propose

$$
\begin{aligned}
u & =\frac{m_{1}}{k_{a}}\left(-\frac{k_{2}}{m_{1}} x_{3}+\frac{\left(k_{1}+k_{2}\right)}{m_{1}} x_{1 r}-\Psi(\cdot)-k_{p 2} e_{3}\right. \\
& \left.-k_{d 2} e_{4}\right)
\end{aligned}
$$

where

$$
\Psi(\cdot)=-\frac{b_{1}}{m_{1}} \dot{x}_{1 r}-\ddot{x}_{1 r}-\frac{k_{2}}{m_{1}} \operatorname{Sat}(\Delta x, d)+\gamma_{1}(\cdot)
$$

is a bounded disturbance.

Substituting (21) in (20), we obtain

$$
\begin{aligned}
& \dot{e_{3}}=e_{4} \\
& \dot{e_{4}}=-\left(\frac{k_{1}+k_{2}}{m_{1}}+k_{p 2}\right) e_{3}-\left(\frac{b_{1}}{m_{1}}+k_{d 2}\right) e_{4} .
\end{aligned}
$$

Since the constants $k_{1}, k_{2}, b_{1}, k_{p 2}$, and $k_{d 2}$ are positive, the origin of (23) is an exponentially stable equilibrium point; as in (23), $k_{p 2}$ and $k_{d 2}$ are control gains to improve the performance of the closed-loop system.

It is important to note that $x_{1}$ converges exponentially to $x_{1 r}$ independent of behavior of $e_{1}$ and $e_{2}$; then $x_{1}$ can be rewritten as

$$
\begin{aligned}
x_{1} & =\frac{m_{2}}{k_{2}}\left(\frac{k_{2}}{m_{2}} r(t)+\frac{b_{2}}{m_{2}} \dot{r}(t)+\ddot{r}(t)-\Gamma(\cdot)-k_{p 1} e_{1}\right. \\
& \left.-k_{d 1} e_{2}\right)+e_{3} .
\end{aligned}
$$

Substituting (24) in (15), we have

$$
\begin{aligned}
& \dot{e_{1}}=e_{2}, \\
& \dot{e_{2}}=-\left(\frac{k_{2}}{m_{2}}+k_{p 1}\right) e_{1}-\left(\frac{b_{2}}{m_{2}}+k_{d 1}\right) e_{2}+\frac{k_{2}}{m_{2}} e_{3},
\end{aligned}
$$


where

$$
\frac{k_{2}}{m_{2}}\left|e_{3}\right| \leq \rho_{0} e^{-\sigma_{0} t},
$$

for some positive constant $\rho_{0}$ and $\sigma_{0}$. Thus, there exist a set of constants $k_{p 1}, k_{p 2}, k_{d 1}$, and $k_{d 2}$ such that the origin of system (25) will be an asymptotically stable equilibrium point in a sufficient large region $\Omega \subset \mathfrak{R}^{4}$.

\section{Control Structure Implementation}

In the previous section, we presented a conceptual solution to the control problem; however, in practice, we cannot implement it directly because the disturbances $\Gamma(\cdot)$ and $\Psi(\cdot)$, as well as signals $x_{4}$ and $e_{4}$, are not available. To solve these problems, in this section, we present the strategy to implement the control input (21) based on the active disturbance rejection control (ADRC) structure, where we use discontinuous state observers to estimate the unknown terms.

To estimate $x_{4}, e_{4}, \Gamma(\cdot)$, and $\Psi(\cdot)$, we propose two robust state observers based on the observer proposed in [26].

For system (20) and the system formed by (7), (8), and (10), we propose the following observers:

$$
\begin{aligned}
\dot{\tilde{e}}_{3}= & \widetilde{e}_{4}+c_{1}\left(y_{e}-\tilde{y}_{e}\right), \\
\dot{\tilde{e}}_{4}= & -\frac{\left(k_{1}+k_{2}\right)}{m_{1}} e_{3}-\frac{b_{1}}{m_{1}} \widetilde{e}_{4}+\frac{k_{2}}{m_{1}} x_{3}-\frac{\left(k_{1}+k_{2}\right)}{m_{1}} x_{1 r} \\
& +\frac{k_{a}}{m_{1}} u+c_{2}\left(y_{e}-\tilde{y}_{e}\right)+c_{3} \operatorname{sign}\left(y_{e}-\tilde{y}_{e}\right), \\
\tilde{y}_{e}= & \tilde{e}_{3} . \\
\dot{\tilde{x}}_{3}= & \tilde{x}_{4}+c_{4}\left(y_{2}-\tilde{y}_{2}\right), \\
\dot{\tilde{x}}_{4}= & \frac{k_{2}}{m_{2}} x_{1}-\frac{k_{2}}{m_{2}} x_{3}-\frac{b_{2}}{m_{2}} \tilde{x}_{4}+c_{5}\left(y_{2}-\tilde{y}_{2}\right) \\
& +c_{6} \operatorname{sign}\left(y_{2}-\tilde{y}_{2}\right), \\
\tilde{y}_{2}= & \tilde{x}_{3}
\end{aligned}
$$

To demonstrate the observer stability, we define error variables $z_{1}=e_{3}-\widetilde{e}_{3}, z_{2}=e_{4}-\widetilde{e}_{4}, z_{3}=x_{3}-\widetilde{x}_{3}$, and $z_{4}=x_{4}-\widetilde{x}_{4}$, whose dynamics are given by

$$
\begin{aligned}
& \dot{z}_{1}=z_{2}-c_{1} z_{1}, \\
& \dot{z}_{2}=-\frac{b_{1}}{m_{1}} z_{2}+\Psi(\cdot)-c_{2} z_{1}-c_{3} \operatorname{sign}\left(z_{1}\right), \\
& \dot{z}_{3}=z_{4}-c_{4} z_{3}, \\
& \dot{z}_{4}=-\frac{b_{2}}{m_{2}} z_{4}+\Gamma(\cdot)-c_{5} z_{3}-c_{6} \operatorname{sign}\left(z_{3}\right),
\end{aligned}
$$

where $\Gamma(\cdot)$ and $\Psi(\cdot)$ are the disturbances that we wish to estimate. Now, with the change of variables $v_{1}=z_{1}, v_{2}=$ $z_{2}-c_{1} z_{1}, v_{3}=z_{3}, v_{4}=z_{4}-c_{4} z_{3}$, we have the systems

$$
\begin{aligned}
\dot{v}_{1}= & v_{2}, \\
\dot{v}_{2}= & -\left(\frac{b_{1}}{m_{1}} c_{1}+c_{2}\right) v_{1}-\left(\frac{b_{1}}{m_{1}}+c_{1}\right) v_{2}-c_{3} \operatorname{sign}\left(v_{1}\right) \\
& +\Psi(\cdot), \\
\dot{v}_{3}= & v_{4}, \\
\dot{v}_{4}= & -\left(\frac{b_{2}}{m_{2}} c_{4}+c_{5}\right) v_{3}-\left(\frac{b_{2}}{m_{2}}+c_{4}\right) v_{4}-c_{6} \operatorname{sign}\left(v_{3}\right) \\
& +\Gamma(\cdot) .
\end{aligned}
$$

According to [26, 27], it is possible to find constants $c_{1}, c_{2}, c_{3}, c_{4}, c_{5}$, and $c_{6}$ such that the origin of the error system will be an asymptotically stable equilibrium point in a region of the state space, where disturbances $\Psi(\cdot)$ and $\Gamma(\cdot)$ are bounded, and we guarantee that $\widetilde{x}_{4}$ and $\widetilde{e}_{4}$ are the estimates of $x_{4}$ and $e_{4}$.

Also, systems (30) and (31) present a second-order sliding mode in $v_{1}=0$ and $v_{3}=0,[26,27]$, where the equivalent control $u_{e q}$ is given by

$$
u_{e q}=\widetilde{\Psi}(\cdot),
$$

for system (30), and

$$
u_{e q}=\widetilde{\Gamma}(\cdot),
$$

for system (31). The equivalent control $u_{e q}$ is the low frequency components of the discontinuous term in the observer when the trajectories are in the sliding surface and we can recover it using a low-pass filter [14]; for example,

$$
F(s)=\frac{\omega_{c}^{2}}{s^{2}+1.4142 \omega_{c} s+\omega_{c}^{2}} .
$$

In this way, the auxiliary reference signal (17) and the control signal (21) are implemented as

$$
\begin{aligned}
x_{1 r} & =\frac{m_{2}}{k_{2}}\left(\frac{k_{2}}{m_{2}} r(t)+\frac{b_{2}}{m_{2}} \dot{r}(t)+\ddot{r}(t)-\widetilde{\Gamma}(\cdot)-k_{p 1} e_{1}\right. \\
& \left.-k_{d 1} \widetilde{e}_{2}\right),
\end{aligned}
$$

where $\tilde{e}_{2}=\tilde{x}_{4}-\dot{r}(t)$ and

$$
\begin{aligned}
u= & \frac{m_{1}}{k_{a}}\left(-\frac{k_{2}}{m_{1}} x_{3}+\frac{\left(k_{1}+k_{2}\right)}{m_{1}} x_{1 r}-\widetilde{\Psi}(\cdot)-k_{p 2} e_{3}\right. \\
& \left.-k_{d 2} \widetilde{e}_{4}\right) .
\end{aligned}
$$

A block diagram of the closed-loop system is shown in Figure 5. 


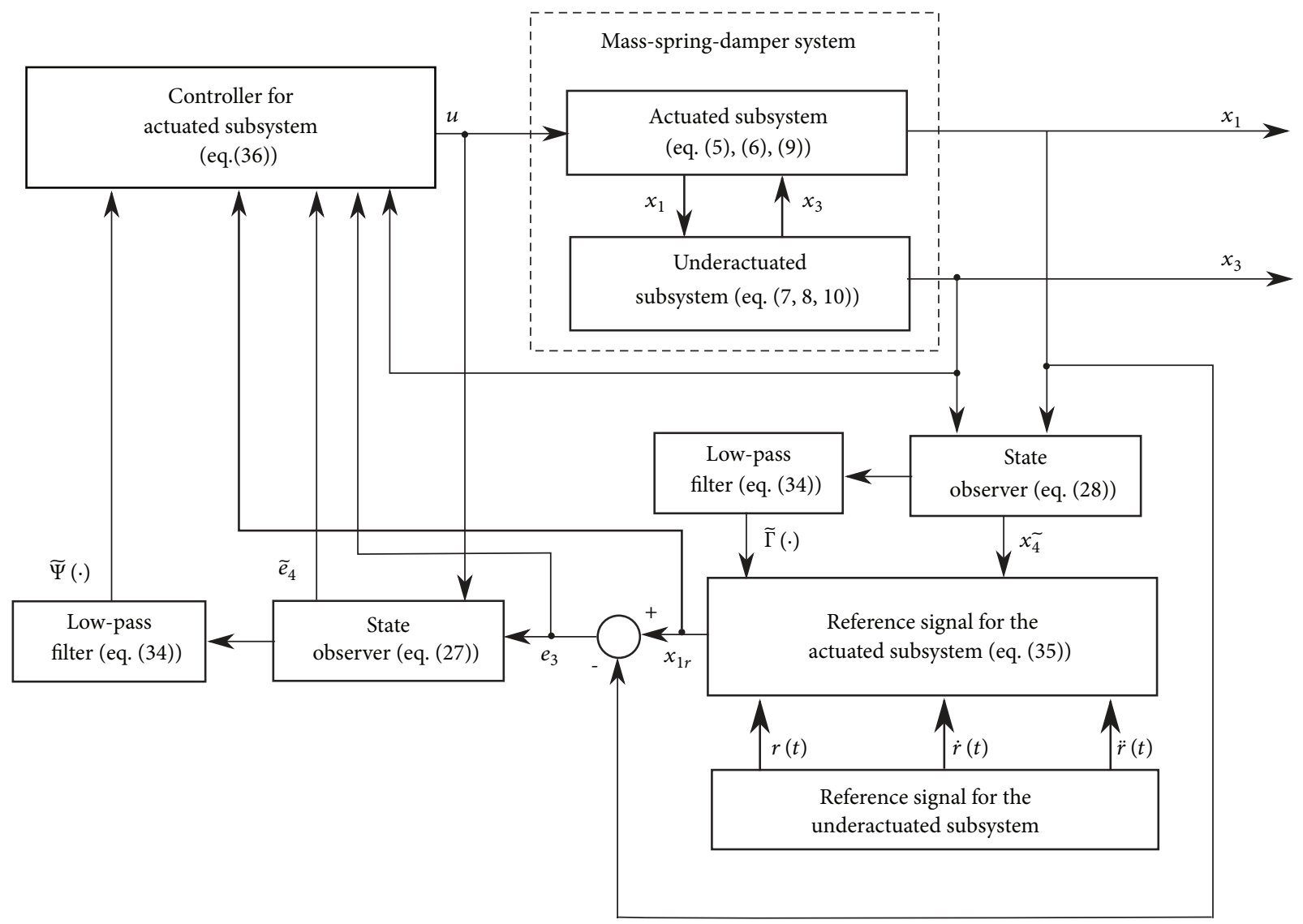

FIGURE 5: A block diagram of the closed-loop system.

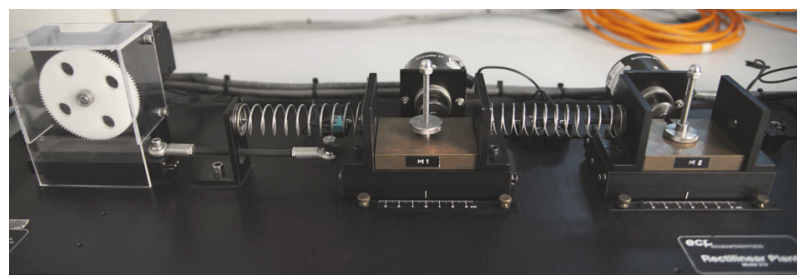

FiguRE 6: 2DOF underactuated mass-spring-damper system with backlash for experiments.

\section{Performance of the Controller}

This section shows the performance of the proposed control strategy on a mass-spring-damper system, model 210, from the Educational Control Products Company shown in Figure 6 . We modified junction between the second spring and the mass $m_{2}$ to introduce backlash (see Figure 7).

In this analysis, we consider two cases of the backlash length $d$; $d=0.001 \mathrm{~m}$ and $d=0.01 \mathrm{~m}$, and the nominal parameters of the system are $m_{1}=m_{2}=0.49 \mathrm{~kg}, k_{1}=$ $121.61 \mathrm{~N} / \mathrm{m}, k_{2}=77.81 \mathrm{~N} / \mathrm{m}, b_{1}=1.73 \mathrm{~kg} / \mathrm{seg}, b_{2}=1.84 \mathrm{~kg} /$ seg, and $k_{m}=1.19 \mathrm{~N} / \mathrm{V}$.

Substituting the nominal parameters, the model of the plant is

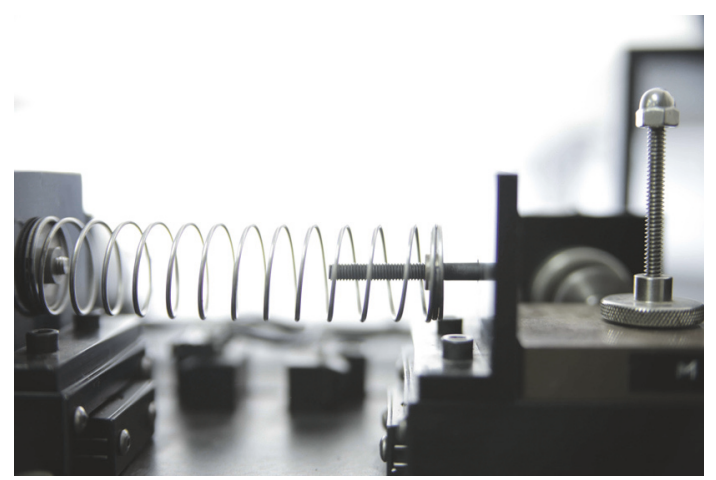

Figure 7: Backlash between second spring $\left(k_{2}\right)$ and second mass $\left(m_{2}\right)$.

$$
\begin{aligned}
\dot{x}_{1}= & x_{2}, \\
\dot{x}_{2}= & -406.98 x_{1}-3.53 x_{2}+158.8 x_{3} \\
& +158.8 S a t(\Delta x, d)+2.43 u+\gamma_{1}(\cdot), \\
\dot{x}_{3}= & x_{4}, \\
\dot{x}_{4}= & 158.8 x_{1}-158.8 x_{3}-3.76 x_{4}-158.8 \operatorname{Sat}(\Delta x, d) \\
& +\gamma_{2}(\cdot) ;
\end{aligned}
$$


meanwhile the state observers (27) and (28) take the following form:

$$
\begin{aligned}
\dot{\tilde{e}}_{3}= & \widetilde{e}_{4}+c_{1}\left(y_{e}-\tilde{y}_{e}\right), \\
\dot{\tilde{e}}_{4}= & -406.98 e_{3}-3.53 \widetilde{e}_{4}+158.8 x_{3}-406.98 x_{1 r} \\
& +2.43 u+c_{2}\left(y_{e}-\tilde{y}_{e}\right)+c_{3} \operatorname{sign}\left(y_{e}-\tilde{y}_{e}\right), \\
\tilde{y}_{e}= & \tilde{e}_{3} . \\
\dot{\tilde{x}}_{3}= & \tilde{x}_{4}+c_{4}\left(y_{2}-\tilde{y}_{2}\right), \\
\dot{\tilde{x}}_{4}= & 158.8 x_{1}-158.8 x_{3}-3.76 \tilde{x}_{4}+c_{5}\left(y_{2}-\tilde{y}_{2}\right) \\
& +c_{6} \operatorname{sign}\left(y_{2}-\tilde{y}_{2}\right), \\
\tilde{y}_{2}= & \tilde{x}_{3}
\end{aligned}
$$

where $c_{1}=50, c_{2}=1, c_{3}=10, c_{4}=50, c_{5}=1$, and $c_{6}=5$, and the constants $c_{3}$ and $c_{6}$ were adjusted considering $d=0.01 \mathrm{~m}$. To estimate the terms $\Gamma(\cdot)$ and $\Psi(\cdot)$, we use the second-order Butterworth low-pass filter (34) with $\omega_{c}=50 \mathrm{rad} / \mathrm{seg}$.

The control signal $x_{1 r}$ to underactuated part of the system takes the following form:

$$
\begin{aligned}
x_{1 r} & =\frac{1}{158.8}\left(-158.8 e_{1}-3.76 \widetilde{e}_{2}-158.8 r(t)\right. \\
& -3.76 \dot{r}(t)-\ddot{r}(t)+\widetilde{\Gamma}(\cdot)),
\end{aligned}
$$

where $e_{1}=x_{3}-r(t)$ and $\tilde{e}_{2}=\tilde{x}_{4}-\dot{r}(t)$. Finally, the control $u$ of the actuated part of the system is

$$
\begin{aligned}
u= & \frac{1}{2.59}\left(158.8 x_{3}-406.98 x_{1 r}-\widetilde{\Psi}(\cdot)-k_{p 2} e_{3}\right. \\
& \left.-k_{d 2} \widetilde{e}_{4}\right),
\end{aligned}
$$

where $e_{3}=x_{1}-x_{1 r}$ and $e_{4}=x_{2}-\dot{x}_{1 r}$.

In the next subsections, we present numerical and experimental results that show the performance of the proposed control strategy. To show the effects of the disturbance compensation in the control system, the simulations and experiments were performed as follows: first, we applied the proposed control in closed loop without compensating the disturbances; in $t=20 \mathrm{sec}$, we added the estimation $\widetilde{\Gamma}(\cdot)$ in the signal $x_{1 r}$ to compensate the term $\Gamma(\cdot)$; finally, in $t=40 \mathrm{sec}$, we added the term $\widetilde{\Psi}(\cdot)$ to control signal $u$ to compensate the term $\Psi(\cdot)$.

5.1. Numerical Results. For numerical simulations, we only present the performance for tracking objective, where the reference signal is $r(t)=0.01 \sin (t)$, while in the subsection of experimental results, we show the performance for tracking and regulation control objectives.

5.1.1. Case 1: Backlash with $d=0.001 \mathrm{~m}$. In Figure 8, we show the performance of the closed-loop system with $d=0.001 \mathrm{~m}$. In the first graph, black line is the reference signal $r(t)$ and the red line is position $x_{3}$. Although the tracking error is little
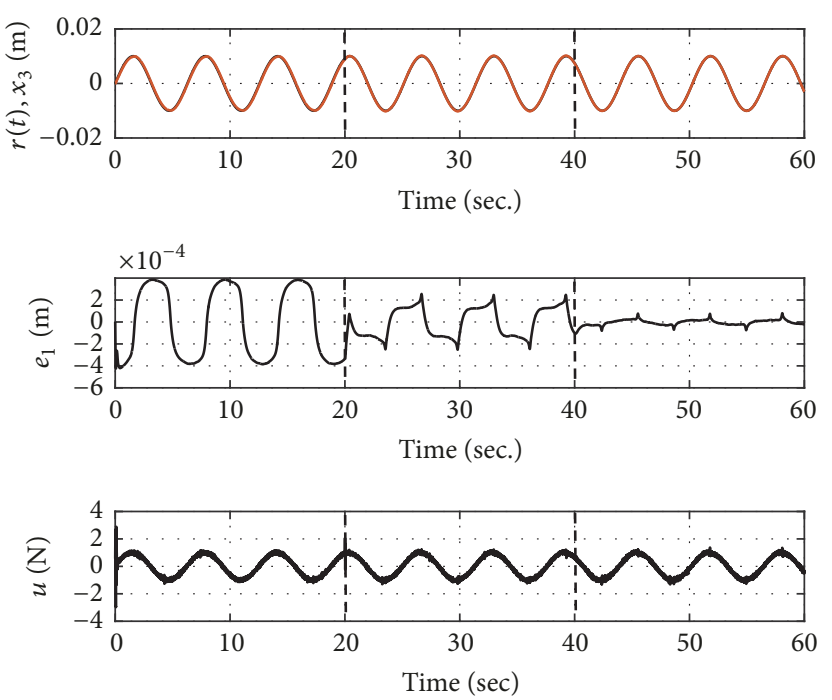

FIGURE 8: Numerical results. Performance of the closed-loop system with $d=0.001 \mathrm{~m}$.

without disturbances compensation, when the compensation is applied, the error is smaller; we achieve an error about $\pm 1 \times 10^{-4} \mathrm{~m}$. It is important to note that control signal has an appropriate range for the experimental implementation of the controller.

5.1.2. Case 2: Backlash with $d=0.01 \mathrm{~m}$. In Figure 9, we show the performance of the closed-loop system with $d=0.01 \mathrm{~m}$. Due to the fact that the backlash is bigger than previous case, the tracking error in closed loop without disturbances compensation is bigger too. When the compensation is made, the error decreases; the tracking error is about $\pm 5 \times$ $10^{-4} \mathrm{~m}$. The control input $u$ has an appropriate range for the experimental implementation of the controller.

5.2. Experimental Results. The experiments were made in a real-time controller dSPACE 1103, using Euler solver with fixed step with sample time $T_{s}=0.00001 \mathrm{sec}$. The parameters of the observers, filters, and constants of the controller are the same as those used in numerical simulation. In this subsection, we present the performance for tracking and regulation control objectives.

5.2.1. Case 1: Backlash with $d=0.001 \mathrm{~m}$. In Figure 10, we show the experimental performance of the closed-loop system with $d=0.001 \mathrm{~m}$. Unlike the simulations, in experiments, the tracking error is big when we do not make the disturbances compensation, but it decreases when they are compensated. We achieve a tracking error about $\pm 5 \times 10^{-4} \mathrm{~m}$, similar to numerical simulations. In this case, we can see clearly that amplitude of the control signal $u$ increases when we add the term $\widetilde{\Psi}(\cdot)$ in $t=40 \mathrm{sec}$.

For regulation, we applied a pulsed signal as a reference with an amplitude of $0.01 \mathrm{~m}$ and a frequency of $0.1 \mathrm{~Hz}$, and the results are shown in Figure 11. When we do not compensate any disturbance, the error between the reference and the output is big. After compensating the disturbances, 

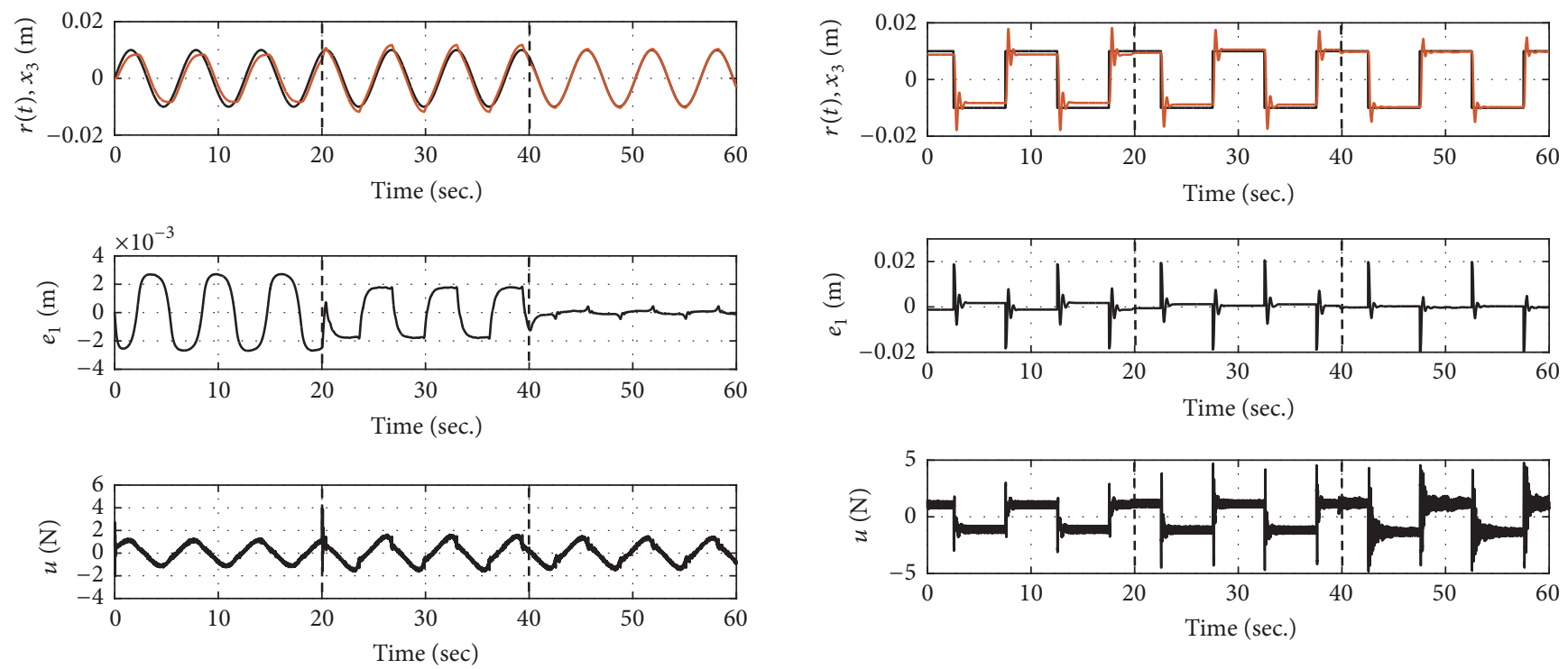

FIGURE 9: Numerical results. Performance of the closed-loop system with $d=0.01 \mathrm{~m}$.
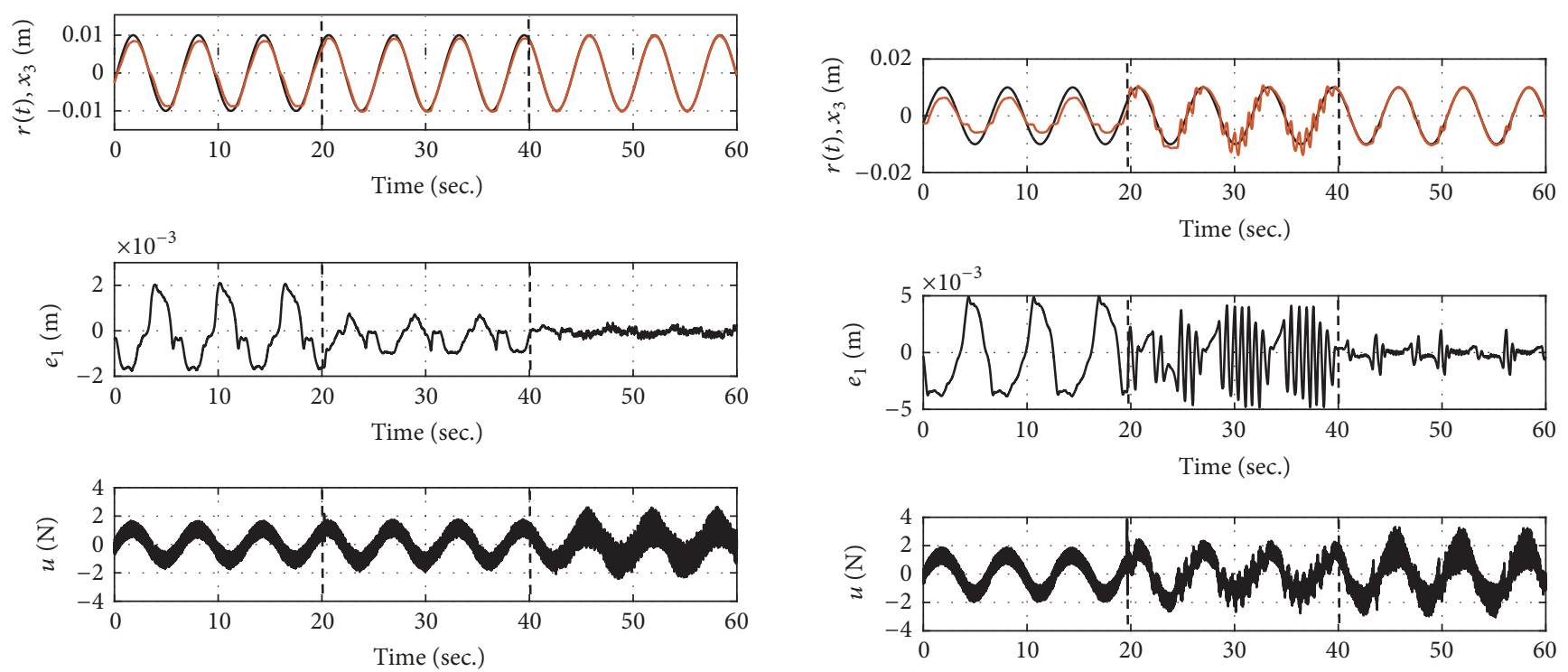

FIGURE 10: Experimental performance of the closed-loop system for tracking and with $d=0.001 \mathrm{~m}$.

we obtain an error about $2.5 \times 10^{-4} \mathrm{~m}$, similar to the tracking case. The control input stays in an adequate range all time.

5.2.2. Case 2: Backlash with $d=0.01 \mathrm{~m}$. In this experiment, we change the parameter $d$ from $0.001 \mathrm{~m}$ to $0.01 \mathrm{~m}$, but the parameters of the observers, filters, and gains of the controller stay without change. For tracking, the results are shown in Figure 12.

In this experiment, we can observe that error $e_{1}$ is very big when we do not compensate the disturbances; it decreases a little when we add the term $\widetilde{\Gamma}(\cdot)$ in $x_{1 r}$, but it decreases considerately when we compensate the term $\widetilde{\Psi}(\cdot)$

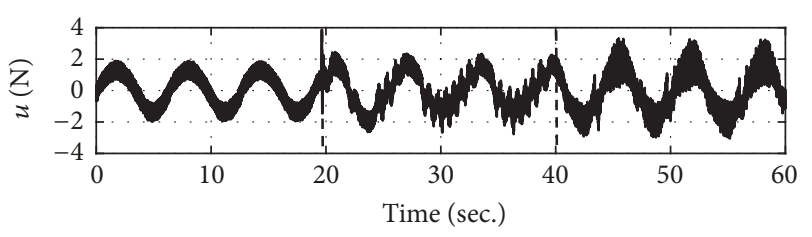

FIGURE 12: Experimental performance of the closed-loop system for tracking and with $d=0.01 \mathrm{~m}$.

in the signal control $u$. We achieve a tracking error about $\pm 2 \times 10^{-3} \mathrm{~m}$. The control signal $u$ stays in the range of $\pm 3 V$.

For regulation objective, we applied the same pulsed signal that we used in Case 1 for experimental results and we obtain similar results as we can see in Figure 13. When we do not compensate any disturbance, the error between the reference and the output is big. After compensating the disturbances, we obtain an error about $1.4 \times 10^{-4} \mathrm{~m}$, similar to the tracking case. The control input stays in an adequate range all time. 

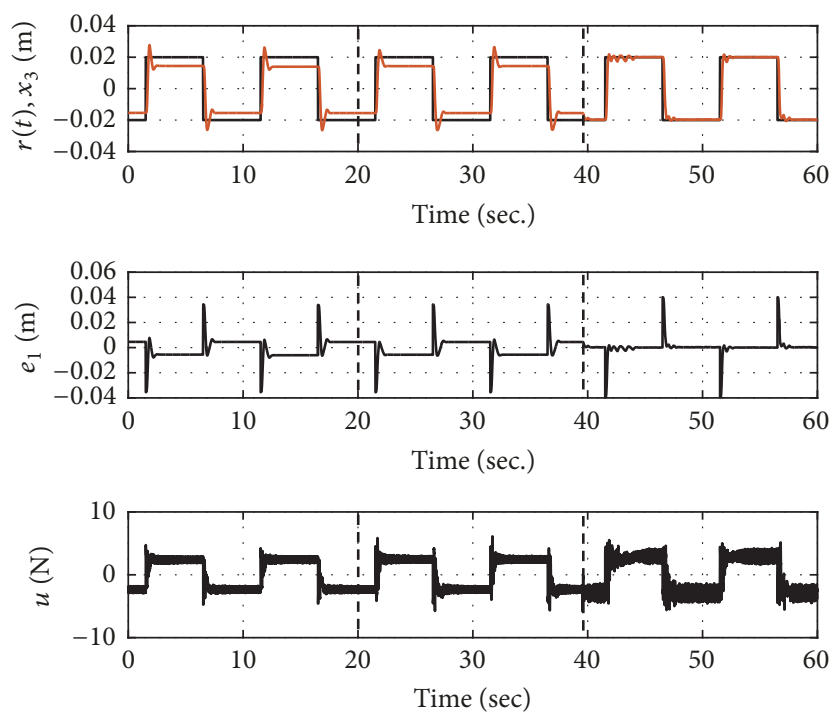

FIGURE 13: Experimental results. Reference signal $r(t)$ (black line) and output $x_{3}$ (red line), case with $d=0.01 \mathrm{~m}$.

\section{Conclusions}

Based on the results of this paper, we can say that the ADRC structure is an adequate option to solve, both theoretically and experimentally, the regulation and tracking control problems on 2DOF underactuated mass-spring-damper system with backlash, uncertainties, and partial measurement of the state vector. The closed-loop system presents good properties of robustness; when the backlash length is $10 \%$ of the maximum amplitude of the reference signal $(d=0.001 \mathrm{~m})$, we achieve a maximum error about $5 \%$ with respect to reference signal; meanwhile when the backlash length is $100 \%$ of the maximum amplitude of the reference signal $(d=0.01 \mathrm{~m})$, we achieve a maximum error about $20 \%$. On the other hand, we can say that the smooth approximation of the backlash, as subtraction of a lineal function and smooth approximation of the saturation function, is a useful model and, therefore, is adequate to solve control problems. A future work is to investigate a broad class of underactuated mechanical systems, where the ADRC structure may solve the regulation and tracking control problems.

\section{Conflicts of Interest}

The authors declare that they have no conflicts of interest.

\section{Acknowledgments}

This research was supported in part by CONACyT, Mexico.

\section{References}

[1] M. W. Spong and D. J. Block, "The Pendubot: A mechatronic system for control research and education," in Proceedings of 1995 34th IEEE Conference on Decision and Control, vol. 1, pp. 555-556, 1995.
[2] M. Spong, "Underactuated mechanical systems," Control problems in robotics and automation, pp. 135-150, 1998.

[3] Y. Liu and H. Yu, "A survey of underactuated mechanical systems," IET Control Theory \& Applications, vol. 7, no. 7, pp. 921-935, 2013.

[4] M. Nordin and P.-O. Gutman, "Controlling mechanical systems with backlash-a survey," Automatica, vol. 38, no. 10, pp. 16331649, 2002.

[5] U. S. Kuruppu, "Hysteresis and backlash-a comparison," International Journal of Control, vol. 2, no. 1, pp. 1-8, 1965.

[6] A. T. Shenton and A. Abass, "Non-parametric linear timeinvariant extensions of non-invertible and backlash plant," International Journal of Robust and Nonlinear Control, vol. 24, no. 18, pp. 3092-3105, 2014.

[7] C.-Y. Su, Y. Stepanenko, J. Svoboda, and T. P. Leung, "Robust adaptive control of a class of nonlinear systems with unknown backlash-like hysteresis," IEEE Transactions on Automatic Control, vol. 45, no. 12, pp. 2427-2432, 2000.

[8] R. Merzouki, J. A. Davila, L. Fridman, and J. C. Cadiou, "Backlash phenomenon observation and identification in electromechanical system," Control Engineering Practice, vol. 15, no. 4, pp. 447-457, 2007.

[9] L. Walha, T. Fakhfakh, and M. Haddar, "Backlash effect on dynamic analysis of a two-stage spur gear system," Journal of Failure Analysis and Prevention (JFAP), vol. 6, no. 3, pp. 60-68, 2006.

[10] O. Begovich, E. N. Sanchez, and M. Maldonado, "TakagiSugeno fuzzy scheme for real-time trajectory tracking of an underactuated robot," IEEE Transactions on Control Systems Technology, vol. 10, no. 1, pp. 14-20, 2002.

[11] M. W. Spong, D. J. Block, and K. J. Astrom, "The mechatronics control kit for education and research," in Proceedings of the IEEE International Conference on Control Applications (CCA'01), pp. 105-110, 2001.

[12] I. Fantoni and R. Lozano, Non-linear control for underactuated mechanical systems, Springer Science \& Business Media, 2002.

[13] K. Lee and V. Coverstone-Carroll, "Control algorithms for stabilizing underactuated robots," Journal of Robotic Systems, vol. 15, no. 12, pp. 681-697, 1998.

[14] V. Utkin, J. Guldner, and J. Shi, Sliding mode control in electromechanical systems, vol. 34, CRC press, 34, 2009.

[15] I. U. Ponce, Y. Orlov, L. T. Aguilar, and J. Alvarez, "Sensorless Nonsmooth Ho-Tracking Synthesis of Servosystems with Backlash and Coulomb Friction," IFAC-PapersOnLine, vol. 48, no. 11, pp. 280-285, 2015.

[16] R. Rascón, J. Alvarez, and L. T. Aguilar, "Discontinuous Ho control of underactuated mechanical systems with friction and backlash," International Journal of Control, Automation and Systems, vol. 14, no. 5, pp. 1213-1222, 2016.

[17] Z. Gao, "On the centrality of disturbance rejection in automatic control," ISA Transactions ${ }^{\circledR}$, vol. 53, no. 4, pp. 850-857, 2014.

[18] J. Q. Han, "From PID to active disturbance rejection control," IEEE Transactions on Industrial Electronics, vol. 56, no. 3, pp. 900-906, 2009.

[19] A. Ferreira, F. J. Bejarano, and L. M. Fridman, "Robust control with exact uncertainties compensation: with or without chattering?" IEEE Transactions on Control Systems Technology, vol. 19, no. 5, pp. 969-975, 2011.

[20] D. I. R. Almeida, J. Alvarez, and J. Peña, "Control structure with disturbance identification for Lagrangian systems," International Journal of Non-Linear Mechanics, vol. 46, no. 3, pp. 486-495, 2011. 
[21] Y. Huang and W. Xue, "Active disturbance rejection control: methodology and theoretical analysis," ISA Transactions ${ }^{\circledR}$, vol. 53, no. 4, pp. 963-976, 2014.

[22] R. Madoński and P. Herman, "Survey on methods of increasing the efficiency of extended state disturbance observers," ISA Transactions $^{\circledR}$, vol. 56, pp. 18-27, 2015.

[23] L. Wang and J. Su, "Disturbance rejection control for nonminimum phase systems with optimal disturbance observer," ISA Transactions ${ }^{\circledR}$, vol. 57, pp. 1-9, 2015.

[24] G. A. Ramos, J. Cortés-Romero, and H. Coral-Enriquez, "Spatial observer-based repetitive controller: An active disturbance rejection approach," Control Engineering Practice, vol. 42, pp. 111, 2015.

[25] S. Oldak, C. Baril, and P. O. Gutman, "Quantitative design of a class of nonlinear systems with parameter uncertainty," International Journal of Robust and Nonlinear Control, vol. 4, no. 1, pp. 101-117, 1994.

[26] D. I. R. Almeida, J. Alvarez, and L. Fridman, "Robust observation and identification of $n$ DOF Lagrangian systems," International Journal of Robust and Nonlinear Control, vol. 17, no. 9, pp. 842-861, 2007.

[27] W. Perruquetti and J. P. Barbot, Sliding Mode Control in Engineering, CRC Press, Boca Raton, Fla, USA, 2002. 


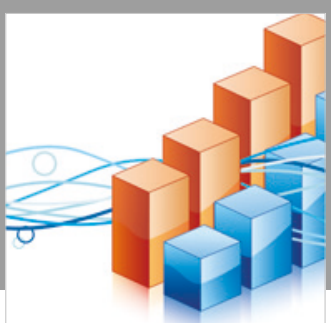

Advances in

Operations Research

\section{-n-m}
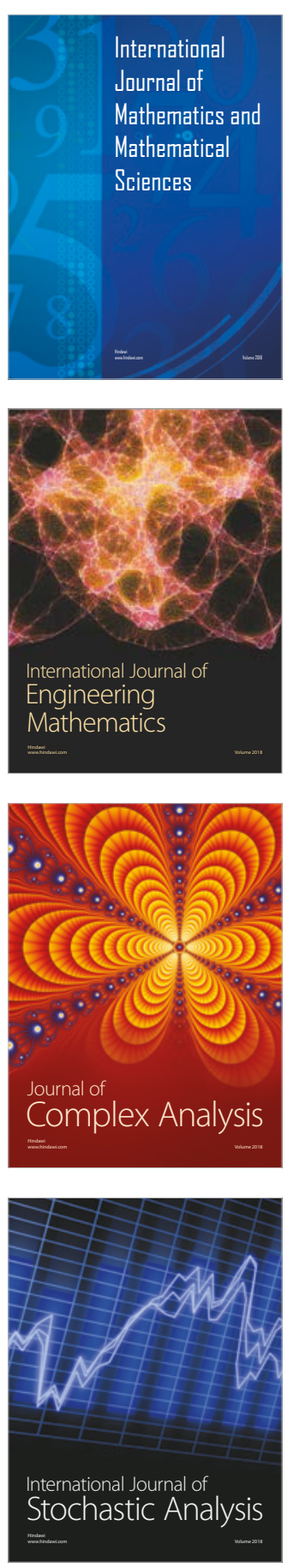
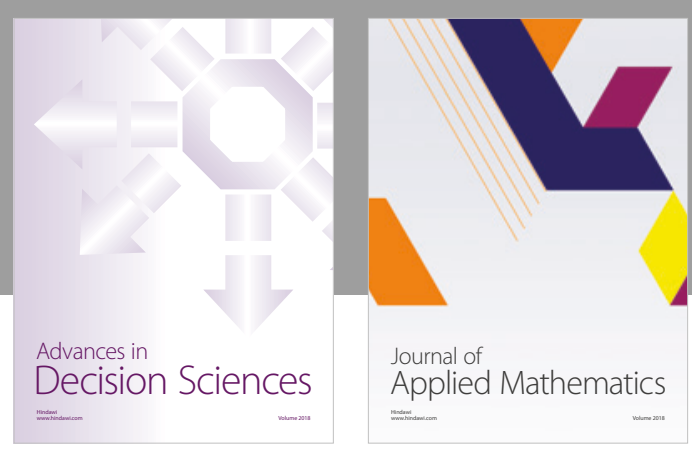

Journal of

Applied Mathematics
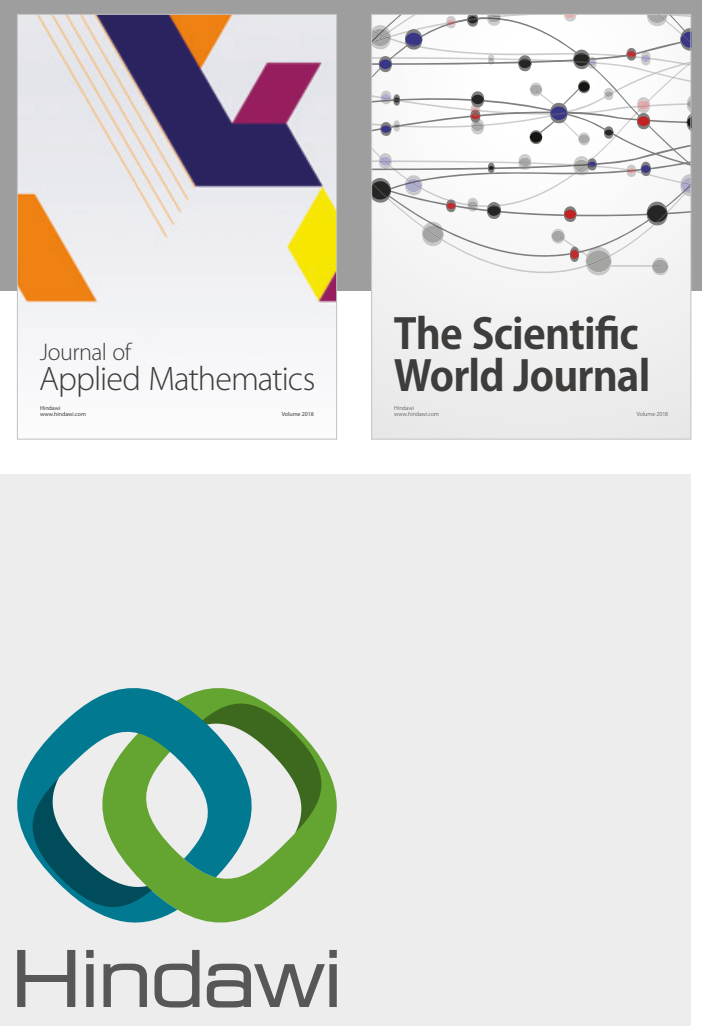

Submit your manuscripts at

www.hindawi.com

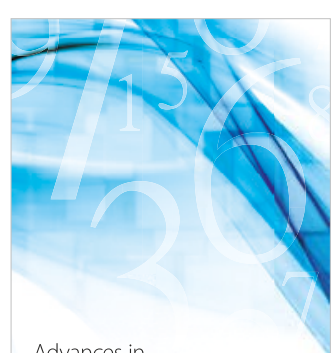

Advances in
Numerical Analysis
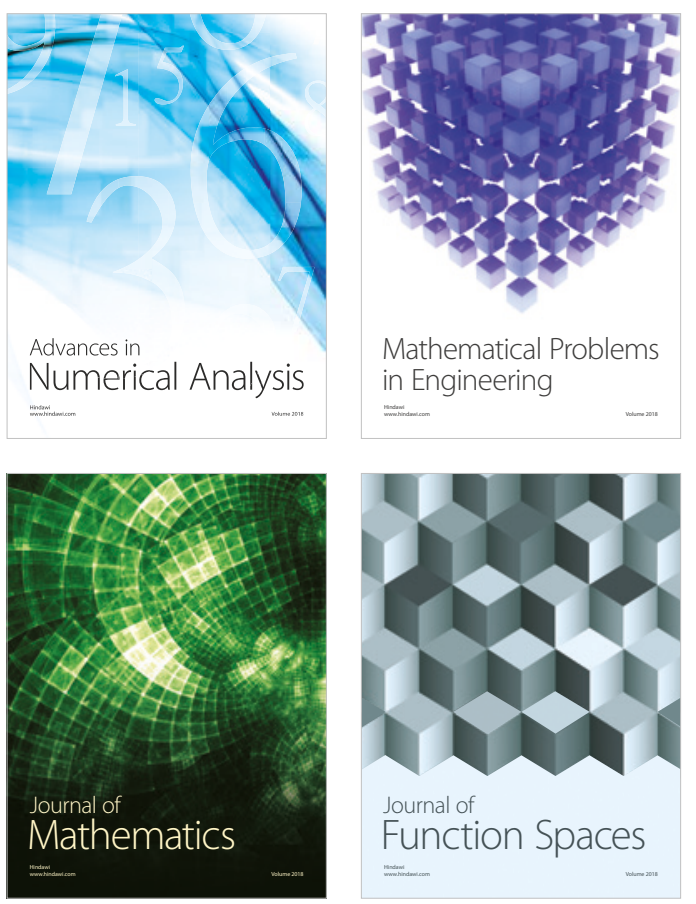

Mathematical Problems in Engineering

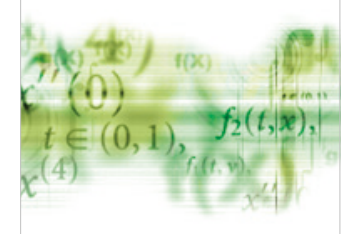

International Journal of

Differential Equations

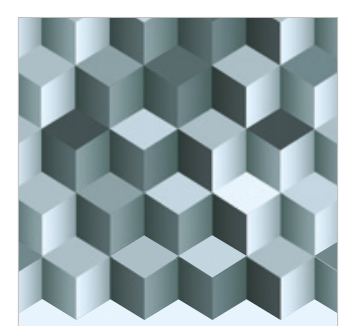

Journal of

Function Spaces

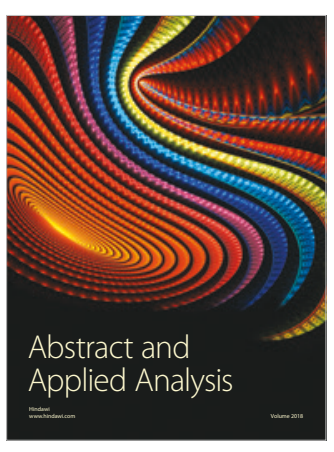

The Scientific

World Journal

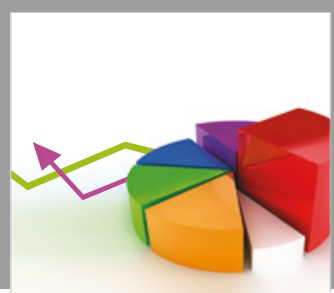

Journal of

Probability and Statistics
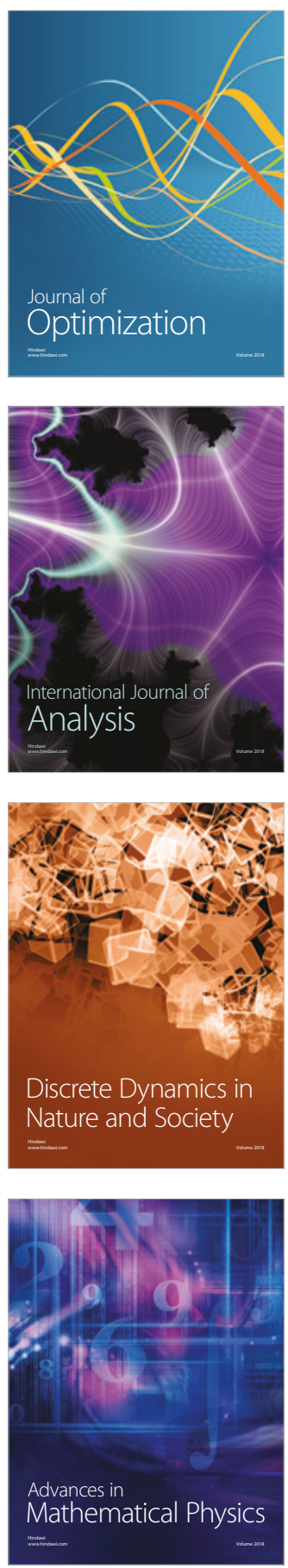